\title{
A Discrete-Time Geo/G/1 Retrial Queue with Two Different Types of Vacations
}

\author{
Feng Zhang and Zhifeng Zhu \\ School of Science, North University of China, Taiyuan 030051, China \\ Correspondence should be addressed to Feng Zhang; gigigi69@163.com \\ Received 9 January 2015; Revised 4 April 2015; Accepted 6 April 2015 \\ Academic Editor: Alexander N. Dudin
}

Copyright (c) 2015 F. Zhang and Z. Zhu. This is an open access article distributed under the Creative Commons Attribution License, which permits unrestricted use, distribution, and reproduction in any medium, provided the original work is properly cited.

\begin{abstract}
We analyze a discrete-time Geo/G/1 retrial queue with two different types of vacations and general retrial times. Two different types of vacation policies are investigated in this model, one of which is nonexhaustive urgent vacation during serving and the other is normal exhaustive vacation. For this model, we give the steady-state analysis for the considered queueing system. Firstly, we obtain the generating functions of the number of customers in our model. Then, we obtain the closed-form expressions of some performance measures and also give a stochastic decomposition result for the system size. Moreover, the relationship between this discrete-time model and the corresponding continuous-time model is also investigated. Finally, some numerical results are provided to illustrate the effect of nonexhaustive urgent vacation on some performance characteristics of the system.
\end{abstract}

\section{Introduction}

During the past few decades, retrial queues have been widely investigated due to their important applications in modeling many practical problems in computer systems, telecommunication networks, and telephone switching systems. In a typical retrial queueing model, an arriving customer who finds the server unavailable may leave the service area and joins a retrial group (called orbit) in order to retry to get the service later after some random time. For more detailed review of the main results and the literature on this topic, the readers are referred to [1-5].

In recent years, there has been a growing interest in studying retrial queueing systems with vacations. According to the vacation policy, the retrial queues with vacation can be divided into two categories: retrial queues with Bernoulli vacation and retrial queues with exhaustive server vacations. Particularly, in retrial queues with Bernoulli vacation, the server will take a single vacation with fixed probability once the service of a customer is finished. Li and Yang [6] firstly studied a retrial queue with a finite number of input sources and Bernoulli vacations. They derived a recursive formula for the steady-state probability and obtained some performance measures of the system by using the method of supplementary variables. Since the work of Li and Yang [6], the retrial queues with Bernoulli vacations have been studied by many authors. For example, Kumar and Arivudainambi [7] considered an $M / G / 1$ retrial queue with Bernoulli vacations and general retrial times. Using matrix-geometric approach, Kumar et al. [8] obtained some performance measures of the $M / M / C$ retrial queue with Bernoulli vacations. In retrial queues with exhaustive vacations, the server can take a vacation when there are no customers in the system. Artalejo [9] firstly analyzed an $M / G / 1$ retrial queue with constant repeated attempts and exhaustive server vacations. $\mathrm{He}$ obtained the probability generating function of the queue size and two stochastic decomposition results for the system size. Later, Aissani [10] generalized the model of Artalejo [9] by considering that the retrial time of a customer follows general distribution. He provided a discrete-event simulation algorithm which can be used to compute the performance of the model. Recently, Chang and Ke [11] and Ke and Chang [12] studied the $M / G / 1$ retrial queues with $J$ vacations. In their model, the server takes at most $J$ vacations until at least one customer is recorded in the orbit as soon as the orbit is empty.

Most of literatures about retrial queues focus on the continuous-time models. In contrast to the continuous case, the discrete-time retrial queues received much less attention 
in literatures. However, the discrete-time retrial models are suitable for the design and analysis of slotted time communication systems such as asynchronous transfer mode (ATM) based systems in broadband integrated services digital network (B-ISDN) and circuit-switched time-division multiple access (TDMA) systems. Yang and Li [13] firstly studied some performance measures of discrete-time $G e o / G / 1$ retrial queues and derived a stochastic decomposition result for the system size. This work was generalized to discrete-time $\mathrm{Geo} / \mathrm{G} / 1$ retrial queue with general retrial times by Atencia and Moreno [14]. The unreliable retrial queues with general retrial times are studied by Wang and Zhao [15] and Atencia et al. [16]. For other literatures concerning discrete-time $\mathrm{Geo} / \mathrm{G} / 1$ retrial queue with general retrial times, see AboulHassan et al. [17, 18], Wu et al. [19], Wang [20], and references therein. Recently, Zhang et al. [21], Yue and Zhang [22], and Zhang and Zhu [23] studied the discrete-time retrial queue with exhaustive vacations. The discrete-time retrial queue with exhaustive single vacations was first studied in Zhang et al. [21]. Then, the model was generalized to the case of exhaustive- $J$ vacations by considering that when the retrial orbit is empty, the server can take at most $J$ vacations continually. In the work of Zhang and Zhu [23], the case that the server may break down is considered based on the model in Yue and Zhang [22].

Most recently, Wu and Yin [24] investigated an unreliable $M / G / 1$ retrial $G$-queue with nonexhaustive random vacation. In contrast to the literatures mentioned above, they assumed that the server takes nonexhaustive random vacations; that is, the server can start a vacation after an exponentially distributed time when the server is busy or idle. To the best of our knowledge, there is no published work on discrete-time Geo/G/1 retrial queue with exhaustive single vacation or nonexhaustive vacations. Although the discrete-time retrial queue with exhaustive random vacations [21-23] has been studied in the past, the case that the server can take nonexhaustive random vacation has not been studied in discrete-time case. In this work, we combine the nonexhaustive random vacation policy and exhaustive single vacation and consider discrete-time retrial queues with two different types of vacations which is very different from the previous work [21-23]. However, it should be pointed out that, in all the aforementioned papers, exhaustive vacation policy is assumed. Our objective in this paper is to study the discrete-time retrial queue with both the no-exhaustive urgent vacation policy and exhaustive single vacation policy. Due to the fact that the no-exhaustive vacation is introduced to the model, new efforts have to be made to overcome the more involved steady-state analysis which include the analysis of the stability of our system and the more involved difference equations. Meanwhile, we prove that there is only one stochastic decomposition property for the system size in contrast to the model [21,23]. So, the analysis in this paper is not a simple repetition of work in [21-23]. This motivates us to investigate such queueing systems in this work.

A possible application of our model is in mobile cellular networks. For an accurate analysis of a mobile cellular network, it can not be ignored that blocked calls are able to redial after some random time [25-27]. In order to simplify the mathematical analysis of the model, we consider only one cell and one channel in the mobile cellular network cellular retrials. If a fresh call is blocked because the channel is unavailable, it enters the retrial orbit and retries to get service after some random length of time. After finishing a call, the base station begins a process of search in order to handle the calls from the retrial orbit in accordance with an FCFS discipline; that is, only the first call in the retrial orbit is permitted to get the service when the channel is available. This kind of retrial policy arises naturally in many practical problems in communication and computer networks where the server is required to search for customers; readers are referred to [28-30]. In order to reduce the energy consumption of the mobile cellular network, the base station can be switched off while there is no call in the retrial orbit. During the period that the base station is switched off, the new arrival fresh calls are deposited in the orbit and the base station will seek to serve the calls from the retrial orbit after the channel is switched on. The period when the base station is switched off may be considered as normal vacation. In addition, if the base station receives an urgent inhibiting signal during serving, it is unavailable for some time and the call being served enters the orbit. This process may be considered as urgent vacation and the calls in the retrial orbit are not allowed for access to the server while the server is on vacations.

The rest of the paper is organized as follows. In Section 2, the description of our model is given. In Section 3, the steady-state analysis for the considered queueing system is presented and the generating functions of the number of customers in the orbit and in the system are obtained. We also obtain the closed-form expressions of some performance measures of the system. We also proved that there is a stochastic decomposition result for the system size in our model. In Section 4, relationship between our model and the continuous-time counterpart is given. Section 5 gives some numerical results to show the effect of some parameters on several performance measures. Finally, conclusions and future research are given in Section 6.

\section{The Mathematical Model}

In this paper, we consider a discrete-time $G e o / G / 1$ retrial queue with two different types of server vacations. It is assumed that the time axis is segmented into a sequence of equal intervals, called slots, and all queueing activities occur at slot boundaries. Let the time axis be marked by $0,1,2, \ldots$. We assume that the departures and the end of the vacations occur in the interval $\left(m^{-}, m\right)$, while arrivals, retrials, and the beginning of the vacations occur in the interval $\left(m, m^{+}\right)$in sequence. The detailed description of our model is given as follows.

Customers arrive according to a geometrical arrival process with parameter $p$, where $p(0<p<1)$ is the probability that an arrival occurs in a slot. There is no waiting space in the system. If an arriving customer finds the server free, the customer is served immediately and leaves the system forever after service completion. Otherwise, if an arriving customer finds that the server is busy or on vacation, in order 
to retry his request after some random time, the retrial time is assumed to follow a general probability distribution $\left\{a_{i}\right\}_{i=0}^{\infty}$ with the generating function $A(x)=\sum_{i=0}^{\infty} a_{i} x^{i}, 0 \leq x \leq 1$.

The service time is assumed to follow a general probability distribution variable $\left\{s_{i}\right\}_{i=1}^{\infty}$ with the generating function $S(x)=\sum_{i=1}^{\infty} s_{i} x^{i}, 0 \leq x \leq 1$, the first moment $S_{1}$, and the second factorial moment $S_{2}=\sum_{i=1}^{\infty} i(i-1) s_{i}$.

It is assumed that the server can take two different types of vacations. The first type of vacation is called nonexhaustive vacation; that is, the server may take an urgent vacation with probability $\bar{\theta}=1-\theta$, when the server is serving a customer, where $\theta$ is the probability that the server does not take urgent vacation. If the server takes urgent vacation, then the customer just being served enters the orbit and the interrupted customer must restart to receive service. The second type of vacation is called normal exhaustive vacation; that is, as soon as the orbit is empty, the server takes a vacation immediately. At the end of both types of vacations, the server becomes idle and waits for serving the customers from outside or orbit.

The urgent vacation time (no-exhaustive vacation) is assumed to follow a general probability distribution $\left\{v_{1, i}\right\}_{i=1}^{\infty}$ with the generating function $V_{1}(x)=\sum_{i=1}^{\infty} v_{1, i} x^{i}, 0 \leq x \leq$ 1 , the first moment $V_{1,1}$, and the second factorial moment $V_{1,2}=\sum_{i=1}^{\infty} i(i-1) v_{1, i}$. The normal vacation time is assumed to follow general distribution $\left\{v_{2, i}\right\}_{i=1}^{\infty}$ with the generating function $V_{2}(x)=\sum_{i=1}^{\infty} v_{2, i} x^{i}, 0 \leq x \leq 1$, the first moment $V_{2,1}$, and the second factorial moment $V_{2,2}=\sum_{i=1}^{\infty} i(i-1) v_{2, i}$.

Finally, we suppose that various stochastic processes involved in the system are assumed to be independent of each other.

\section{The Steady-State Analysis}

In this section, we will show the steady-state analysis for the considered queueing system. Firstly, the Markov chain underlying the considered queueing system and Kolmogorov equations of the steady-state probabilities are obtained. Then, we derive the generating functions of the numbers of customers of the system. Finally, some performance measures are given.

3.1. Markov Chain and Steady-State Equations. At time $m^{+}$, let $C_{m}$ be the state of the server, $C_{m}=0,1,2$ or 3 according to whether the server is free, busy, on urgent vacation, or on normal vacation, and let $N_{m}$ be the number of the customers in the orbit. If $C_{m}=0, \xi_{m}$ represents the remaining retrial time. If $C_{m}=1, \xi_{m}$ represents the remaining service time of the customer currently being served. If $C_{m}=2, \xi_{m}$ represents the remaining urgent vacation time. If $C_{m}=3, \xi_{m}$ represents the remaining normal vacation time. Thus, at time $m^{+}$, the system can be described by the process $Y_{m}=\left(C_{m}, \xi_{m}, N_{m}\right)$. It can be shown that $\left\{Y_{m}, m=0,1,2, \ldots\right\}$ is a Markov chain with the following state space:

$$
\begin{gathered}
\Omega=\{(0,0)\} \cup\{(0, i, k): i \geq 1, k \geq 1\} \\
\cup\{(1, i, k): i \geq 1, k \geq 0\}
\end{gathered}
$$

$$
\begin{aligned}
& \cup\{(2, i, k): i \geq 1, k \geq 1\} \\
& \cup\{(3, i, k): i \geq 1, k \geq 0\} .
\end{aligned}
$$

Firstly, we define the stationary probabilities of the Markov chain $\left\{Y_{m}, m=0,1,2, \ldots\right\}$ as follows:

$$
\begin{aligned}
\pi_{0,0} & =\lim _{m \rightarrow \infty} P\left\{C_{m}=0, N_{m}=0\right\}, \\
\pi_{0, i, k} & =\lim _{m \rightarrow \infty} P\left\{C_{m}=0, \xi_{m}=i, N_{m}=k\right\} ;
\end{aligned}
$$$$
i \geq 1, k \geq 1
$$$$
\pi_{1, i, k}=\lim _{m \rightarrow \infty} P\left\{C_{m}=1, \xi_{m}=i, N_{m}=k\right\} ;
$$$$
i \geq 1, k \geq 0 \text {, }
$$

$\pi_{2, i, k}=\lim _{m \rightarrow \infty} P\left\{C_{m}=2, \xi_{m}=i, N_{m}=k\right\} ;$

$i \geq 1, k \geq 1$,

$\pi_{3, i, k}=\lim _{m \rightarrow \infty} P\left\{C_{m}=3, \xi_{m}=i, N_{m}=k\right\} ;$

$i \geq 1, k \geq 0$.

Then, the Kolmogorov equations are obtained as follows:

$$
\begin{aligned}
& \pi_{0,0}=\bar{p} \pi_{0,0}+\bar{p} \pi_{3,1,0}, \\
& \pi_{0, i, k}=\bar{p} \pi_{0, i+1, k}+\bar{p} a_{i} \pi_{1,1, k}+\bar{p} a_{i} \pi_{2,1, k}+\bar{p} a_{i} \pi_{3,1, k}, \\
& i \geq 1, k \geq 1,
\end{aligned}
$$

$$
\begin{aligned}
\pi_{1, i, k}= & \delta_{0, k} p \theta s_{i} \pi_{0,0}+\left(1-\delta_{0, k}\right) p \theta s_{i} \sum_{j=1}^{\infty} \pi_{0, j, k} \\
& +\bar{p} \theta s_{i} \pi_{0,1, k+1}+p \theta s_{i} \pi_{1,1, k}+\bar{p} a_{0} \theta s_{i} \pi_{1,1, k+1} \\
& +\left(1-\delta_{0, k}\right) p \theta \pi_{1, i+1, k-1}+\bar{p} \theta \pi_{1, i+1, k} \\
& +\bar{p} a_{0} \theta s_{i} \pi_{2,1, k+1}+\left(1-\delta_{0, k}\right) p \theta s_{i} \pi_{2,1, k} \\
& +p \theta s_{i} \pi_{3,1, k}+\bar{p} a_{0} \theta s_{i} \pi_{3,1, k+1}, \quad i \geq 1, k \geq 0 \\
\pi_{2, i, k}= & \delta_{1, k} p \bar{\theta} v_{1, i} \pi_{0,0}+\left(1-\delta_{1, k}\right) p \bar{\theta} v_{1, i} \sum_{j=1}^{\infty} \pi_{0, j, k-1} \\
& +\bar{p} \bar{\theta} v_{1, i} \pi_{0,1, k}+p \bar{\theta} v_{1, i} \pi_{1,1, k-1} \\
& +\bar{p} a_{0} \bar{\theta} v_{1, i} \pi_{1,1, k}+\left(1-\delta_{1, k}\right) p \pi_{2, i+1, k-1} \\
& +\bar{p} \pi_{2, i+1, k}+\bar{p} a_{0} \bar{\theta} v_{1, i} \pi_{2,1, k} \\
& +\left(1-\delta_{1, k}\right) p \bar{\theta} v_{1, i} \pi_{2,1, k-1}+p \bar{\theta} v_{1, i} \pi_{3,1, k-1} \\
& +\bar{p} a_{0} \bar{\theta} v_{1, i} \pi_{3,1, k}+\left(1-\delta_{1, k}\right) p \bar{\theta} v_{1, i} \sum_{j=2}^{\infty} \pi_{1, j, k-2} \sum_{j=2}^{\infty} \pi_{1, j, k-1}, \quad i \geq 1, k \geq 1, \\
& +k
\end{aligned}
$$




$$
\begin{aligned}
\pi_{3, i, k}= & \bar{p} \pi_{3, i+1, k}+\left(1-\delta_{0, k}\right) p \pi_{3, i+1, k-1} \\
& +\delta_{0, k} \bar{p} v_{2, i} \pi_{1,1,0}, \quad i \geq 1, \quad k \geq 0 .
\end{aligned}
$$

The normalizing condition is

$$
\pi_{0,0}+\sum_{i=1}^{\infty} \sum_{k=1}^{\infty}\left(\pi_{0, i, k}+\pi_{2, i, k}\right)+\sum_{i=1}^{\infty} \sum_{k=0}^{\infty}\left(\pi_{1, i, k}+\pi_{3, i, k}\right)=1
$$

where $\delta_{0, k}=1$, if $k=0$; otherwise, $\delta_{0, k}=0$ if $k \neq 0$.

3.2. The Generating Functions. To solve (3)-(8), we introduce the generating functions

$$
\begin{aligned}
& \varphi_{0}(x, z)=\sum_{i=1}^{\infty} \sum_{k=1}^{\infty} \pi_{0, i, k} x^{i} z^{k}, \\
& \varphi_{1}(x, z)=\sum_{i=1}^{\infty} \sum_{k=0}^{\infty} \pi_{1, i, k} x^{i} z^{k}, \\
& \varphi_{2}(x, z)=\sum_{i=1}^{\infty} \sum_{k=1}^{\infty} \pi_{2, i, k} x^{i} z^{k}, \\
& \varphi_{3}(x, z)=\sum_{i=1}^{\infty} \sum_{k=0}^{\infty} \pi_{3, i, k} x^{i} z^{k}
\end{aligned}
$$

and the auxiliary generating functions

$$
\begin{array}{ll}
\varphi_{0, i}(z)=\sum_{k=1}^{\infty} \pi_{0, i, k} z^{k}, & i \geq 1, \\
\varphi_{1, i}(z)=\sum_{k=0}^{\infty} \pi_{1, i, k} z^{k}, & i \geq 1,
\end{array}
$$

$$
\begin{gathered}
\varphi_{2, i}(z)=\sum_{k=1}^{\infty} \pi_{2, i, k} z^{k}, \quad i \geq 1, \\
\varphi_{3, i}(z)=\sum_{k=0}^{\infty} \pi_{3, i, k} z^{k}, \quad i \geq 1 .
\end{gathered}
$$

Now, we can solve (3)-(8) by using the generating function technique. We first give some lemmas which will be used later on and their proof which can be readily obtained. Thus, they are omitted here.

Lemma 1. The following inequalities hold:

$$
\begin{array}{ll}
S(x) \leq x & \text { for } 0 \leq x \leq 1 \\
V_{i}(x) \leq x & \text { for } i=1,2,0 \leq x \leq 1 .
\end{array}
$$

Lemma 2. If $p \theta+\bar{\theta}+\bar{\theta} p V_{1,1}<\rho_{1}$, then the inequality

$$
[z+(1-z) \bar{p} A(\bar{p})] \Omega(z)-z \gamma(z)(1-\theta \gamma(z))>0
$$

holds for $0 \leq z<1$, where

$$
\begin{aligned}
\gamma(z)= & \bar{p}+p z \\
\rho_{1}= & \frac{\bar{\theta}(p+\bar{p} A(\bar{p}))}{1-S(\theta)}, \\
\Omega(z)= & (1-\theta \gamma(z)) S(\theta \gamma(z)) \\
& +z[1-S(\theta \gamma(z))] \bar{\theta} V_{1}(\gamma(z)) .
\end{aligned}
$$

Lemma 3. If $p \theta+\bar{\theta}+\bar{\theta} p V_{1,1}<\rho_{1}$, the following limits exist:

$$
\begin{aligned}
\lim _{z \rightarrow 1} \frac{C(z)(1-\theta \gamma(z))}{\Lambda(z)} & =\frac{\bar{\theta}\left[p\left(1-V_{2,1}\right)-A(\bar{p})\left(p+V_{2}(\bar{p})\right)\right]}{(1-S(\theta))\left(p \theta+\bar{\theta}+p V_{1,1} \bar{\theta}\right)-\bar{\theta}(p+\bar{p} A(\bar{p}))} \\
\lim _{z \rightarrow 1} \frac{(1-\theta \gamma(z)) \Gamma(z)-\left(p+V_{2}(\bar{p})\right) \Omega(z)}{\Lambda(z)} & =\frac{\bar{\theta}\left[p\left(1+V_{2}(\bar{p})\right)-p \bar{p} V_{2,1}\right]+\left(p+V_{2}(\bar{p})\right)(1-S(\theta))\left(p \theta+\bar{\theta}+p V_{1,1} \bar{\theta}\right)}{(1-S(\theta))\left(p \theta+\bar{\theta}+p V_{1,1} \bar{\theta}\right)-\bar{\theta}(p+\bar{p} A(\bar{p}))}
\end{aligned}
$$

where

$$
\begin{aligned}
\Gamma(z)= & \gamma(z)\left(1+V_{2}(\bar{p})\right)-\bar{p} V_{2}(\gamma(z)), \\
C(z)= & z\left(\gamma(z)-V_{2}(\gamma(z))\right)+A(\bar{p})(1-z) \Gamma(z), \\
\Lambda(z)= & {[z+\bar{p} A(\bar{p})(1-z)] \Omega(z) } \\
& -z \gamma(z)(1-\theta \gamma(z)) .
\end{aligned}
$$

By using Lemmas 1-3, we can obtain the generating functions of the stationary distribution of the system which are given by the following theorem.
Theorem 4. If $p \theta+\bar{\theta}+\bar{\theta} p V_{1,1}<\rho_{1}$, the stationary distribution of the Markov chain $\left\{Y_{m}, m=0,1,2, \ldots\right\}$ has the following generating functions:

$$
\begin{aligned}
& \varphi_{0}(x, z)=\frac{A(x)-A(\bar{p})}{x-\bar{p}} \\
& . \frac{(1-\theta \gamma(z)) \Gamma(z)-\left(p+V_{2}(\bar{p})\right) \Omega(z)}{\Lambda(z) V_{2}(\bar{p})} p x z \pi_{0,0} \\
& \varphi_{1}(x, z)=\frac{S(x)-S(\theta \gamma(z))}{x-\theta \gamma(z)} \frac{C(z)(1-\theta \gamma(z))}{\Lambda(z) V_{2}(\bar{p})} \\
& \cdot p x \theta \pi_{0,0},
\end{aligned}
$$




$$
\begin{aligned}
\varphi_{2}(x, z) & =\frac{V_{1}(x)-V_{1}(\gamma(z))}{x-\gamma(z)} \frac{C(z)[1-S(\theta \gamma(z))]}{\Lambda(z) V_{2}(\bar{p})} \\
\cdot p x \bar{\theta} z \pi_{0,0}, & \\
\varphi_{3}(x, z) & =\frac{p x\left[V_{2}(x)-V_{2}(\gamma(z))\right]}{[x-\gamma(z)] V_{2}(\bar{p})} \pi_{0,0},
\end{aligned}
$$

where

$$
\begin{aligned}
& \pi_{0,0}=\frac{(1-S(\theta))\left(p \theta+\bar{\theta}+p V_{1,1} \bar{\theta}\right)-\bar{\theta}(p+\bar{p} A(\bar{p}))}{\bar{\theta} S(\theta)\left[p\left(1-V_{2,1}\right)-A(\bar{p})\left(p+V_{2}(\bar{p})\right)\right]} \\
& \cdot V_{2}(\bar{p}) .
\end{aligned}
$$

Proof. Multiplying (4)-(7) by $z^{k}$, and summing over $k$, we get the following equations:

$$
\begin{aligned}
& \varphi_{0, i}(z) \\
& =\bar{p} \varphi_{0, i+1}(z)+\bar{p} a_{i}\left(\varphi_{1,1}(z)+\varphi_{2,1}(z)+\varphi_{3,1}(z)\right) \\
& \quad-\bar{p} a_{i}\left(\pi_{1,1,0}+\pi_{3,1,0}\right), \quad i \geq 1, \\
& \varphi_{1, i}(z) \\
& =p \theta s_{i} \pi_{0,0}+p \theta s_{i} \varphi_{0}(1, z)+\frac{\bar{p}}{z} \theta s_{i} \varphi_{0,1}(z) \\
& \quad+(\bar{p}+p z) \theta \varphi_{1, i+1}(z) \\
& \quad+\left(p+\frac{\bar{p} a_{0}}{z}\right) \theta s_{i}\left(\varphi_{1,1}(z)+\varphi_{2,1}(z)+\varphi_{3,1}(z)\right) \\
& \quad-\frac{\bar{p}}{z} a_{0} \theta s_{i}\left(\pi_{1,1,0}+\pi_{3,1,0}\right), \quad i \geq 1,
\end{aligned}
$$

$\varphi_{2, i}(z)$

$$
\begin{aligned}
&= p z \bar{\theta} v_{1, i} \pi_{0,0}+p z \bar{\theta} v_{1, i} \varphi_{0}(1, z)+\bar{p} \bar{\theta} v_{1, i} \varphi_{0,1}(z) \\
&+(\bar{p}+p z) \varphi_{2, i+1}(z) \\
&+\left(p z+\bar{p} a_{0}\right) \bar{\theta} v_{1, i}\left(\varphi_{1,1}(z)+\varphi_{2,1}(z)+\varphi_{3,1}(z)\right) \\
&+z \gamma(z) \bar{\theta} v_{1, i} \varphi_{1,1}(z)-\bar{p} a_{0} \bar{\theta} s_{2, i}\left(\pi_{1,1,0}+\pi_{3,1,0}\right), \\
& i \geq 1, \\
& \varphi_{3, i}(z)=(\bar{p}+p z) \varphi_{3, i+1}(z)+\bar{p} v_{2, i} \pi_{1,1,0}, \quad i \geq 1 .
\end{aligned}
$$

Multiplying (21) by $x^{i}$, summing over $i$, and letting $\gamma(z)=$ $\bar{p}+p z$, we get

$$
\frac{x-\gamma(z)}{x} \varphi_{3}(x, z)=-\gamma(z) \varphi_{3,1}(z)+\bar{p} V_{2}(x) \pi_{1,1,0} .
$$

Setting $x=\gamma(z)$ in (22), we get

$$
\varphi_{3,1}(z)=\frac{\bar{p} V_{2}(\gamma(z))}{\gamma(z)} \pi_{1,1,0} .
$$

Substituting (23) into (22), we obtain

$$
\varphi_{3}(x, z)=\frac{\bar{p} x\left[V_{2}(x)-V_{2}(\gamma(z))\right]}{x-\gamma(z)} \pi_{1,1,0} .
$$

Differentiating (24) with respect to $x$ and setting $x=z=0$, we get

$$
\pi_{3,1,0}=V_{2}(\bar{p}) \pi_{1,1,0} .
$$

Substituting (25) into (3), we get

$$
\pi_{1,1,0}=\frac{p \pi_{0,0}}{\bar{p} V_{2}(\bar{p})} .
$$

Multiplying (18)-(20) by $x^{i}$, summing over $i$, and using (23), (25), and (26), we obtain that

$$
\begin{aligned}
& \frac{x-\bar{p}}{x} \varphi_{0}(x, z)=\bar{p}\left(A(x)-a_{0}\right)\left(\varphi_{1,1}(z)+\varphi_{2,1}(z)\right) \\
& -\bar{p} \varphi_{0,1}(z)-p\left(A(x)-a_{0}\right) \\
& \cdot \frac{\gamma(z)\left(1+V_{2}(\bar{p})\right)-\bar{p} V_{2}(\gamma(z))}{V_{2}(\bar{p}) \gamma(z)} \pi_{0,0}, \\
& \frac{x-\gamma(z) \theta}{x} \varphi_{1}(x, z)=\left[\frac{p z+\bar{p} a_{0}}{z} \theta S(x)-\theta \gamma(z)\right] \\
& \cdot \varphi_{1,1}(z)+\frac{\bar{p}}{z} \theta S(x) \varphi_{0,1}(z)+\frac{p z+\bar{p} a_{0}}{z} \theta S(x) \\
& \cdot \varphi_{2,1}(z)+p \theta S(x) \varphi_{0}(1, z) \\
& +\frac{K(z)-a_{0} \gamma(z)\left(1+V_{2}(\bar{p})\right)}{z V_{2}(\bar{p}) \gamma(z)} p \theta S(x) \pi_{0,0}, \\
& \frac{x-\gamma(z)}{x} \varphi_{2}(x, z)=\left[\left(\bar{p} a_{0}+p z\right) \bar{\theta} V_{1}(x)-\gamma(z)\right] \\
& \cdot \varphi_{2,1}(z)+\bar{p} \bar{\theta} V_{1}(x) \varphi_{0,1}(z) \\
& +\left[\left(p z+\bar{p} a_{0}\right)-\left(p z^{2}+\bar{p} z\right)\right] \bar{\theta} V_{1}(x) \varphi_{1,1}(z) \\
& +p z \bar{\theta} V_{1}(x) \varphi_{0}(1, z)+z(p z+\bar{p}) \bar{\theta} V_{1}(x) \varphi_{1}(1, z) \\
& +\frac{K(z)-a_{0} \gamma(z)\left(1+V_{2}(\bar{p})\right)}{V_{2}(\bar{p}) \gamma(z)} p \bar{\theta} V_{1}(x) \pi_{0,0},
\end{aligned}
$$

where $K(z)=z \gamma(z) V_{2}(\bar{p})+\left(p z+\bar{p} a_{0}\right) V_{2}(\gamma(z))$.

In order to find $\varphi_{0}(1, z)$ in (28), we set $x=1$ in (27) and get

$$
\begin{aligned}
p \varphi_{0}(1, z) & \\
= & \bar{p}\left(1-a_{0}\right)\left(\varphi_{1,1}(z)+\varphi_{2,1}(z)\right)-\bar{p} \varphi_{0,1}(z) \\
& \quad-p\left(1-a_{0}\right) \frac{\gamma(z)\left(1+V_{2}(\bar{p})\right)-\bar{p} V_{2}(\gamma(z))}{V_{2}(\bar{p}) \gamma(z)} \pi_{0,0} .
\end{aligned}
$$


Substituting (30) into (28), we get

$$
\begin{aligned}
& \frac{x-\gamma(z) \theta}{x} \varphi_{1}(x, z) \\
& =\left[\frac{z+\bar{p} a_{0}(1-z)}{z} \theta S(x)-\theta \gamma(z)\right] \varphi_{1,1}(z) \\
& +\frac{\bar{p}(1-z)}{z} \theta S(x) \varphi_{0,1}(z)+\frac{z+\bar{p} a_{0}(1-z)}{z} \theta S(x) \\
& \cdot \varphi_{2,1}(z)-\frac{z\left[\gamma(z)-V_{2}(\gamma(z))\right]+a_{0}(1-z) \Gamma(z)}{z V_{2}(\bar{p}) \gamma(z)} \\
& \cdot p \theta S(x) \pi_{0,0} .
\end{aligned}
$$

Note that, by setting $x=1$ in (31), we can get $\varphi_{1}(1, z)$; then substituting $\varphi_{0}(1, z)$ and $\varphi_{1}(1, z)$ into $(29)$, we get

$$
\begin{aligned}
& \frac{x-\gamma(z)}{x} \varphi_{2}(x, z)=\left[\frac{z+\bar{p} a_{0}(1-z)}{1-\theta \gamma(z)} \bar{\theta} V_{1}(x)-\gamma(z)\right] \\
& \cdot \varphi_{2,1}(z)+\frac{\bar{p}(1-z)}{1-\theta \gamma(z)} \bar{\theta} V_{1}(x) \varphi_{0,1}(z) \\
& +\frac{\left(p z+\bar{p} a_{0}\right)(1-z)}{1-\theta \gamma(z)} \bar{\theta} V_{1}(x) \varphi_{1,1}(z) \\
& -\frac{z\left[\gamma(z)-V_{2}(\gamma(z))\right]+a_{0}(1-z) \Gamma(z)}{V_{2}(\bar{p}) \gamma(z)(1-\theta \gamma(z))} p \bar{\theta} V_{1}(x) \\
& \cdot \pi_{0,0},
\end{aligned}
$$

where $\Gamma(z)=\gamma(z)\left(1+V_{2}(\bar{p})\right)-\bar{p} V_{2}(\gamma(z))$.

Setting $x=\bar{p}$ in (27), $x=\theta \gamma(z)$ in (31), and $x=\gamma(z)$ in (32), respectively, we can get the equations for $\varphi_{0,1}(z), \varphi_{1,1}(z)$, and $\varphi_{2,1}(z)$. By solving these equations, we get the generating functions as follows:

$$
\begin{gathered}
\varphi_{0,1}(z)=p z\left(A(\bar{p})-a_{0}\right) \\
\cdot \frac{(1-\theta \gamma(z)) \Gamma(z)-\left(p+V_{2}(\bar{p})\right) \Omega(z)}{\Lambda(z) V_{2}(\bar{p})} \frac{\pi_{0,0}}{\bar{p}}, \\
\varphi_{1,1}(z)=\frac{C(z)(1-\theta \gamma(z)) p S(\theta \gamma(z))}{\Lambda(z) V_{2}(\bar{p}) \gamma(z)} \pi_{0,0}, \\
\varphi_{2,1}(z)=\frac{C(z) z[1-S(\theta \gamma(z))] p \bar{\theta} V_{1}(\gamma(z))}{\Lambda(z) V_{2}(\bar{p}) \gamma(z)} \pi_{0,0} .
\end{gathered}
$$

Using Lemmas 1-3, it is easy to show that $\varphi_{0,1}(z), \varphi_{1,1}(z)$, and $\varphi_{2,1}(z)$ are defined for $z \in[0,1)$ and can be extended by continuity in $z=1$, if $p \theta+\bar{\theta}+\bar{\theta} p V_{1,1}<\rho_{1}$. Now substituting (26) into (24) we can get $\varphi_{3}(x, z)$. Similarly, substituting (33) into (27), (31) and (32), we obtain $\varphi_{0}(x, z), \varphi_{1}(x, z)$, and $\varphi_{2}(x, z)$. Using the normalizing condition, we can find the unknown constant $\pi_{0,0}$. This completes the proof.

3.3. Performance Measures. In this subsection, we give some performance measures based on Theorem 4 . The results are summarized in the following corollary and the proof is omitted here. For convenience, we define variable $N$ as the orbit size and $L$ as the system size.

Corollary 5. (1) The marginal generating function of the number of customers in the orbit when the server is idle or on vacation is given by

$$
\begin{aligned}
\pi_{0,0}+\varphi_{0}(1, z)+\varphi_{2}(1, z)+\varphi_{3}(1, z) \\
=\frac{[(p \theta+\bar{\theta}) S(\theta \gamma(z))-p \theta z] C(z)}{\Lambda(z) V_{2}(\bar{p})} \pi_{0,0} .
\end{aligned}
$$

(2) The marginal generating function of the number of customers in the orbit when the server is busy is given by

$$
\varphi_{1}(1, z)=\frac{[1-S(\theta \gamma(z))] C(z)}{\Lambda(z) V_{2}(\bar{p})} p \theta \pi_{0,0} .
$$

(3) The marginal generating function of the number of customers in the orbit when the server is on urgent vacation is given by

$$
\begin{aligned}
& \varphi_{2}(1, z) \\
& \quad=\frac{1-V_{1}(\gamma(z))}{1-z} \frac{C(z)[1-S(\theta \gamma(z))]}{\Lambda(z) V_{2}(\bar{p})} \bar{\theta} z \pi_{0,0} .
\end{aligned}
$$

(4) The marginal generating function of the number of customers in the orbit when the server is on vacation is given by

$$
\varphi_{3}(1, z)=\frac{1-V_{2}(\gamma(z))}{(1-z) V_{2}(\bar{p})} \pi_{0,0} .
$$

(5) The generating function of the number of customers in the orbit is given by

$$
\begin{aligned}
\Psi(z)= & \pi_{0,0}+\varphi_{0}(1, z)+\varphi_{1}(1, z)+\varphi_{2}(1, z) \\
& +\varphi_{3}(1, z) \\
= & \frac{[\bar{\theta} S(\theta \gamma(z))+p \theta(1-z)] C(z)}{\Lambda(z) V_{2}(\bar{p})} \pi_{0,0} .
\end{aligned}
$$

(6) The probability generating function of the number of customers in the system is given by

$$
\begin{aligned}
\Phi(z)= & \pi_{0,0}+\varphi_{0}(1, z)+z \varphi_{1}(1, z)+\varphi_{2}(1, z) \\
& +\varphi_{3}(1, z) \\
= & \frac{[\bar{\theta}+p \theta(1-z)] S(\theta \gamma(z)) C(z)}{\Lambda(z) V_{2}(\bar{p})} \pi_{0,0} .
\end{aligned}
$$

Corollary 6. (1) The probability that system is empty is

$$
\begin{aligned}
& \pi_{0,0}^{\pi_{0}}=\frac{(1-S(\theta))\left(p \theta+\bar{\theta}+p V_{1,1} \bar{\theta}\right)-\bar{\theta}(p+\bar{p} A(\bar{p}))}{\bar{\theta} S(\theta)\left[p\left(1-V_{2,1}\right)-A(\bar{p})\left(p+V_{2}(\bar{p})\right)\right]} \\
& \quad \cdot V_{2}(\bar{p}) .
\end{aligned}
$$


(2) The probability that the server is idle is

$$
\begin{aligned}
\pi_{0,0} & +\varphi_{0}(1,1)+\varphi_{2}(1,1)+\varphi_{3}(1,1) \\
& =1-\frac{p \theta(1-S(\theta))}{\bar{\theta} S(\theta)} .
\end{aligned}
$$

(3) The probability that the server is busy is

$$
\varphi_{1}(1,1)=\frac{p \theta(1-S(\theta))}{\bar{\theta} S(\theta)} .
$$

(4) The probability that the server is on urgent vacation is

$$
\varphi_{2}(1,1)=\frac{p V_{1,1}(1-S(\theta))}{S(\theta)} .
$$

(5) The probability that the server is on normal vacation is $\varphi_{3}(1,1)=p V_{2,1}$

$$
\frac{(1-S(\theta))\left(p \theta+\bar{\theta}+p V_{1,1} \bar{\theta}\right)-\bar{\theta}(p+\bar{p} A(\bar{p}))}{\bar{\theta} S(\theta)\left[p\left(1-V_{2,1}\right)-A(\bar{p})\left(p+V_{2}(\bar{p})\right)\right]} \text {. }
$$

(6) The mean number of customers in the orbit is

$$
\begin{aligned}
E(N)=\left.\Psi^{\prime}(z)\right|_{z=1}= & \frac{2 p \theta\left(\bar{\theta} S^{\prime}(\theta)-1\right)\left[p\left(1-V_{2,1}\right)-A(\bar{p})\left(p+V_{2}(\bar{p})\right)\right]+2 \bar{\theta} S(\theta) \mathbf{O}-p^{2} V_{2,2}}{2 \bar{\theta} S(\theta)\left[p\left(1-V_{2,1}\right)-A(\bar{p})\left(p+V_{2}(\bar{p})\right)\right]} \\
& -\frac{\bar{\theta}(1-S(\theta)) p^{2} V_{1,2}+2[1-\bar{p} A(\bar{p})] \mathbf{P}+2 \mathbf{Q}}{2(1-S(\theta))\left(p \theta+\bar{\theta}+p V_{1,1} \bar{\theta}\right)-\bar{\theta}(p+\bar{p} A(\bar{p}))} .
\end{aligned}
$$

(7) The mean number of customers in the system is

$$
\begin{aligned}
E(L)=\left.\Phi^{\prime}(z)\right|_{z=1}= & \frac{2 p \theta\left(\bar{\theta} S^{\prime}(\theta)-1\right)\left[p\left(1-V_{2,1}\right)-A(\bar{p})\left(p+V_{2}(\bar{p})\right)\right]+2 \bar{\theta} S(\theta) \mathbf{O}-p^{2} V_{2,2}}{2 \bar{\theta} S(\theta)\left[p\left(1-V_{2,1}\right)-A(\bar{p})\left(p+V_{2}(\bar{p})\right)\right]}+\frac{p \theta(1-S(\theta))}{\bar{\theta} S(\theta)} \\
& -\frac{\bar{\theta}(1-S(\theta)) p^{2} V_{1,2}+2[1-\bar{p} A(\bar{p})] \mathbf{P}+2 \mathbf{Q}}{2(1-S(\theta))\left(p \theta+\bar{\theta}+p V_{1,1} \bar{\theta}\right)-\bar{\theta}(p+\bar{p} A(\bar{p}))}
\end{aligned}
$$

where

$$
\begin{aligned}
\mathbf{O}= & p\left(1-V_{2,1}\right)-A(\bar{p})\left(p+p V_{2}(\bar{p})-p \bar{p} V_{2,1}\right) \\
\mathbf{P}= & \bar{\theta}\left(1+p V_{1,1}\right)(1-S(\theta))-p \theta S(\theta) \\
\mathbf{Q}= & \bar{\theta}(1-S(\theta)) p V_{1,1}-2 p \theta S^{\prime}(\theta)\left(p \theta+\bar{\theta}+p V_{1,1} \bar{\theta}\right) \\
& -p(\bar{\theta}-\theta-p \theta) .
\end{aligned}
$$

Remark 7. Consider some special cases.

(i) When $a_{0}=1$, that is, the case of no retrial, $\Phi(z)$ reduces to

$$
\begin{aligned}
\Phi(z) \\
=\frac{[\bar{\theta}+p \theta(1-z)] S(\theta \gamma(z))\left[\bar{\theta}-(1-S(\theta))\left(p \theta+\bar{\theta}+p V_{1,1} \bar{\theta}\right)\right]}{\Omega(z)-z(1-\theta \gamma(z))} \\
\\
\quad \times \frac{1+V_{2}(\bar{p})(1-z)-V_{2}(\gamma(z))}{p V_{1}+V_{2}(\bar{p})},
\end{aligned}
$$

which is the generating function of the number of customers in the orbit for the $\mathrm{Geo} / \mathrm{G} / 1$ queue with two different types of vacations without retrial customers. (ii) When $\theta=1$, that is, the case of no urgent vacation, $\Phi(z)$ reduces to

$$
\begin{aligned}
\Phi(z) & \\
= & \frac{C(z)}{[z+\bar{p} A(\bar{p})(1-z)] S(\bar{p}+p z)-z(\bar{p}+p z)} \\
& \cdot \frac{p+\bar{p} A(\bar{p})-p S_{1}}{\left(p+V_{2}(\bar{p})\right) A(\bar{p})+p V_{2,1}-p},
\end{aligned}
$$

which is the generating function of the number of customers in the orbit for the discrete-time $G e o / G / 1$ general retrial queue with single vacation. The stochastic decomposition property for the system size was proposed by Fuhrmann and Cooper [31] in the queueing systems with vacations. The property of stochastic decomposition can be applied to study the proximity between the steady-state distribution for the standard queueing system without vacations and the corresponding queueing system with vacations. Artalejo and Falin [32] obtained a similar stochastic decomposition result for a retrial queue. In this section, we obtain the property of stochastic decomposition of the system size for our model, which is given by the following theorem and we find that there 
is only one stochastic decomposition property for the system size in contrast to the model $[21,23]$.

Theorem 8. The total number of customers $(L)$ in the system can be decomposed as the sum of two independent random variables $L=L_{1}+M_{1} . L_{1}$ is the number of customers in the Geo/G/1/queue with urgent vacation and $M_{1}$ is the number of repeated customers given that the server is idle or on normal vacation.

Proof. After some algebra operation, $\Phi(z)$ can be expressed by

$$
\Phi(z)=\Phi_{1}(z) \Phi_{2}(z)
$$

where

$$
\begin{aligned}
& \Phi_{1}(z) \\
& =\frac{(1-\theta \gamma(z)) S(\theta \gamma(z))(1-z)}{(1-\theta \gamma(z))[S(\theta \gamma(z))-z]+z[1-S(\theta \gamma(z))] \bar{\theta} V_{1}(\gamma(z))} \\
& \quad \times \frac{\bar{\theta} S(\theta)-(1-S(\theta))\left[p \theta+p \bar{\theta} V_{1,1}\right]}{\bar{\theta} S(\theta)}
\end{aligned}
$$

is the generating function of $G e o / G / 1$ queue with urgent vacation and $\Phi_{2}(z)$ is given by

$$
\begin{aligned}
& \Phi_{2}(z) \\
& =\frac{(1-\theta \gamma(z))[S(\theta \gamma(z))-z]+z[1-S(\theta \gamma(z))] \bar{\theta} V_{1}(\gamma(z))}{(1-z) \Lambda(z) V_{2}(\bar{p})} \\
& \quad \cdot \pi_{0,0} \times \frac{\bar{\theta} S(\theta) C(z)}{\bar{\theta} S(\theta)-(1-S(\theta))\left[p \theta+p \bar{\theta} V_{1,1}\right]} .
\end{aligned}
$$

It is easy to verify that

$$
\Phi_{2}(z)=\frac{\pi_{0,0}+\varphi_{0}(1,1)+\varphi_{3}(1, z)}{\pi_{0,0}+\varphi_{0}(1,1)+\varphi_{3}(1,1)},
$$

which is the generating function given that the server is idle or on normal vacation.

In order to prove that $\Phi_{1}(z)$ is the generating function of $G e o / G / 1$ queue with urgent vacation, we use the same notations as in the previous sections; then we can get the following equations:

$$
\begin{aligned}
\pi_{0,0}= & \bar{p} \pi_{0,0}+\bar{p} \pi_{1,1,0}, \\
\pi_{1, i, k}= & \delta_{0, k} p \theta s_{i} \pi_{0,0}+p \theta s_{i} \pi_{1,1, k}+\bar{p} \theta s_{i} \pi_{1,1, k+1} \\
& +\left(1-\delta_{0, k}\right) p \theta \pi_{1, i+1, k-1}+\bar{p} \theta \pi_{1, i+1, k} \\
& +\bar{p} \theta s_{i} \pi_{2,1, k+1}+\left(1-\delta_{0, k}\right) p \theta s_{i} \pi_{2,1, k} \\
& \quad i \geq 1, k \geq 0,
\end{aligned}
$$

$$
\begin{aligned}
\pi_{2, i, k}= & \delta_{1, k} p \bar{\theta} v_{1, i} \pi_{0,0}+p \bar{\theta} v_{1, i} \pi_{1,1, k-1}+\bar{p} \bar{\theta} v_{1, i} \pi_{1,1, k} \\
& +\left(1-\delta_{1, k}\right) p \pi_{2, i+1, k-1}+\bar{p} \pi_{2, i+1, k} \\
& +\bar{p} \bar{\theta} v_{1, i} \pi_{2,1, k}+\left(1-\delta_{1, k}\right) p \bar{\theta} v_{1, i} \pi_{2,1, k-1} \\
& +\left(1-\delta_{1, k}\right) p \bar{\theta} v_{1, i} \sum_{j=2}^{\infty} \pi_{1, j, k-2} \\
& +\bar{p} \bar{\theta} v_{1, i} \sum_{j=2}^{\infty} \pi_{1, j, k-1} \quad i \geq 1, k \geq 1
\end{aligned}
$$

with the normalizing condition

$$
\pi_{0,0}+\sum_{i=1}^{\infty} \sum_{k=0}^{\infty} \pi_{1, i, k}+\sum_{i=1}^{\infty} \sum_{k=1}^{\infty} \pi_{2, i, k}=1
$$

Solving the above equations, we can get that the generating function of the system size is

$$
\begin{aligned}
& \Phi_{1}(z) \\
& =\frac{(1-\theta \gamma(z)) S(\theta \gamma(z))(1-z)}{(1-\theta \gamma(z))[S(\theta \gamma(z))-z]+z[1-S(\theta \gamma(z))] \bar{\theta} V_{1}(\gamma(z))} \\
& \quad \times \frac{\bar{\theta} S(\theta)-(1-S(\theta))\left[p \theta+p \bar{\theta} V_{1,1}\right]}{\bar{\theta} S(\theta)} .
\end{aligned}
$$

This completes the proof.

\section{Relation to Corresponding Continuous-Time Model}

In this section, we prove that our model can be used to approximate the corresponding continuous-time $M / G / 1$ general retrial queueing system with two different types of vacations. For this continuous-time model, we give the corresponding assumption. Specifically it is assumed that the arrival process of customers is a Poisson process with rate $\lambda$. If an arriving customer finds the server free, he begins his service immediately. Otherwise, if the server is busy or on vacation, the customer joins the orbit. Once the system becomes empty, the server takes a normal exhaustive single vacation immediately. In addition, the server may take an urgent vacation with probability $\bar{\theta}=1-\theta$ when the server is serving a customer. The retrial times, the service times, the urgent vacation times, and the normal vacation times have general distributions denoted by $R(x), S(x), V_{1}(x)$, and $V_{2}(x)$, respectively, and their Laplace-Stieltjes transforms are denoted by $\widetilde{R}(s), \widetilde{S}(s), \widetilde{V_{1}}(s)$, and $\widetilde{V_{2}}(s)$, respectively, and their finite means are denoted by $R_{1}, S_{1}$ and $V_{1,1}$ and $V_{2,1}$, respectively.

Suppose that the time is divided into intervals of equal length $\Delta$. Using the technique given by Yang and $\mathrm{Li}$ [13], the continuous-time $M / G / 1$ retrial queue model can be approximated by a discrete-time model. 
Let

$$
\begin{aligned}
p & =\lambda \Delta, \\
a_{i} & =\int_{i \Delta}^{(i+1) \Delta} d R(x), \\
s_{i} & =\int_{(i-1) \Delta}^{i \Delta} d S_{l}(x), \\
v_{l, i} & =\int_{(i-1) \Delta}^{i \Delta} d V_{l}(x), \quad l=1,2 .
\end{aligned}
$$

By using the definition of Lebesgue integration, we can get the following results:

$$
\lim _{\Delta \rightarrow 0} p V_{i, 1}=\lambda V_{i, 1}, \quad i=1,2,
$$

$$
\begin{aligned}
\lim _{\Delta \rightarrow 0} S(\theta \gamma(z)) & =\widetilde{S}[\lambda(1-z)+\theta], \\
\lim _{\Delta \rightarrow 0} \rho & =\lambda E(S), \\
\lim _{\Delta \rightarrow 0} V_{i}[\gamma(z)] & =\widetilde{V}_{i}[\lambda(1-z)], \quad i=1,2, \\
\lim _{\Delta \rightarrow 0} V_{2}(\bar{p}) & =\widetilde{V}_{2}(\lambda), \\
\lim _{\Delta \rightarrow 0} A(\bar{p}) & =A(\lambda) .
\end{aligned}
$$

From the above relations, we obtain the generating functions of the number of customers in the orbit for the corresponding continuous-time $M / G / 1$ retrial queue model with two different types of vacations which is given as follows:

$$
\begin{aligned}
\lim _{\Delta \rightarrow 0} \Phi(z)= & \frac{z\left(1-\widetilde{V_{2}}[\lambda(1-z)]\right)+\widetilde{R}(\lambda)(1-z)\left[1+\widetilde{V_{2}}(\lambda)-\widetilde{V_{2}}(\lambda(1-z))\right]}{\left[\omega(z) \widetilde{S}[\omega(z)]+z[1-\widetilde{S}[\omega(z)]] \theta \widetilde{V_{1}}[\lambda(1-z)]\right][\widetilde{R}(\lambda)(1-z)+z]-z \omega(z)} \\
& \times \frac{\theta \widetilde{R}(\lambda)-[1-\widetilde{S}(\theta)]\left[\lambda+\theta+\lambda V_{1,1} \theta\right]}{\left[\lambda V_{2,1}+\widetilde{R}(\lambda) \widetilde{V_{2}}(\lambda)\right] \theta \widetilde{S}(\theta)} \omega(z) \widetilde{S}[\omega(z)],
\end{aligned}
$$

where $\omega(z)=\theta+\lambda(1-z)$.

\section{Numerical Results}

In this section the results of some numerical examples are given to illustrate the effect of some parameters on the characteristics of the system. Specifically we consider two performance measures: the probability that the system is empty $\pi_{0,0}$ and the mean orbit size $E(N)$.

We assume that the retrial times, the service time, the urgent vacation, and the normal vacation time are all geometric distributions with parameters $r, \mu, v_{1}$, and $v_{2}$, respectively. Their generating functions are given by

$$
\begin{aligned}
A(x) & =\frac{1-r}{1-r x}, \\
S(x) & =\frac{(1-\mu) x}{1-\mu x}, \\
V_{1}(x) & =\frac{\left(1-v_{1}\right) x}{1-v_{1} x}, \\
V_{2}(x) & =\frac{\left(1-v_{2}\right) x}{1-v_{2} x}
\end{aligned}
$$

for $0 \leq x \leq 1$.

For convenience, we choose the arrival rate $p=0.2$, the retrial rate $r=0.1$, and the service rate $\mu=0.3$ in all the numerical examples. In Figures 1 and 2 , the probability $\pi_{0,0}$ that the system is empty is plotted against the parameter $\theta$, where $\theta$ is the probability that the server does not take urgent vacation. We choose $V_{1,1}=5 / 4,5 / 3$, and 2 and $V_{2,1}=2$ in
Figure 1, and we choose $V_{2,1}=2,5$, and 10 in Figure 2. From Figures 1 and 2, we observe that $\pi_{0,0}$ increases significantly with parameter $\theta$.

For different values of the mean urgent vacation time $V_{1,1}$, we observe that $\pi_{0,0}$ decreases with increasing values of $V_{1,1}$ in Figure 1. As the mean urgent vacation time increases, the expected waiting time for a customer increases, and therefore the probability that the system is empty $\pi_{0,0}$ decreases. In Figure 2 , it is observed that $\pi_{0,0}$ decreases with the increasing values of $V_{2,1}$.

In Figures 3 and 4 , the mean number $E(N)$ of customers in the orbit is plotted against the parameter $\theta$. Specifically, we choose $V_{1,1}=5 / 4,5 / 3$, and 2 in Figure 3, and we choose $V_{2,1}=$ 2, 5, and 10 in Figure 4. From Figures 3 and 4, we observe that $E(N)$ exhibits a stable decreasing with the increasing of parameter $\theta$. In addition, we can observe in Figure 3 that $E(N)$ increases with $V_{1,1}$. As the mean urgent vacation time increases, the mean sojourn time for a customer increases and therefore the number of customers in the system increases. Moreover, the parameter $\theta$ affects $E(N)$ more apparently when the value of the parameter $\theta$ is getting small. In other words, the parameter $\theta$ hardly affects $E(N)$ when $\theta$ goes to 1 .

It is observed in Figure 4 that the mean orbit size $E(N)$ also increases with increasing values of $V_{1,1}$. Moreover, when $\theta$ is getting small the parameter $\theta$ affects $E(N)$ more apparently.

\section{Conclusions and Future Research}

In this work, we study discrete-time retrial queues with two different types of vacations in which the server can 


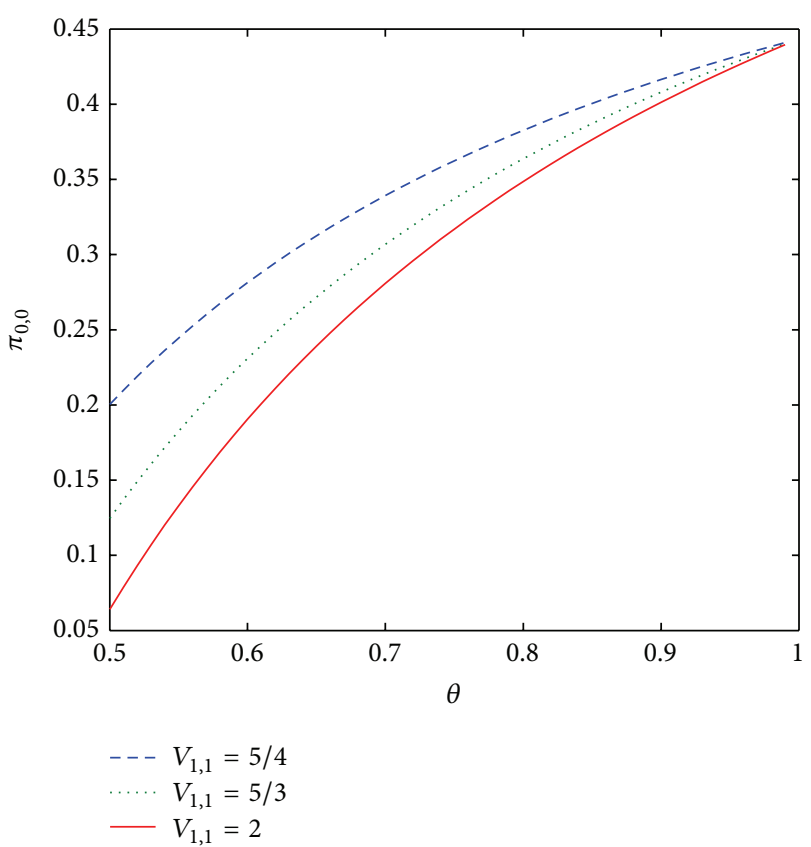

FIGURE $1: \pi_{0,0}$ versus $\theta$ for $p=0.2, r=0.1, \mu=0.3$, and $v_{2}=0.5$.

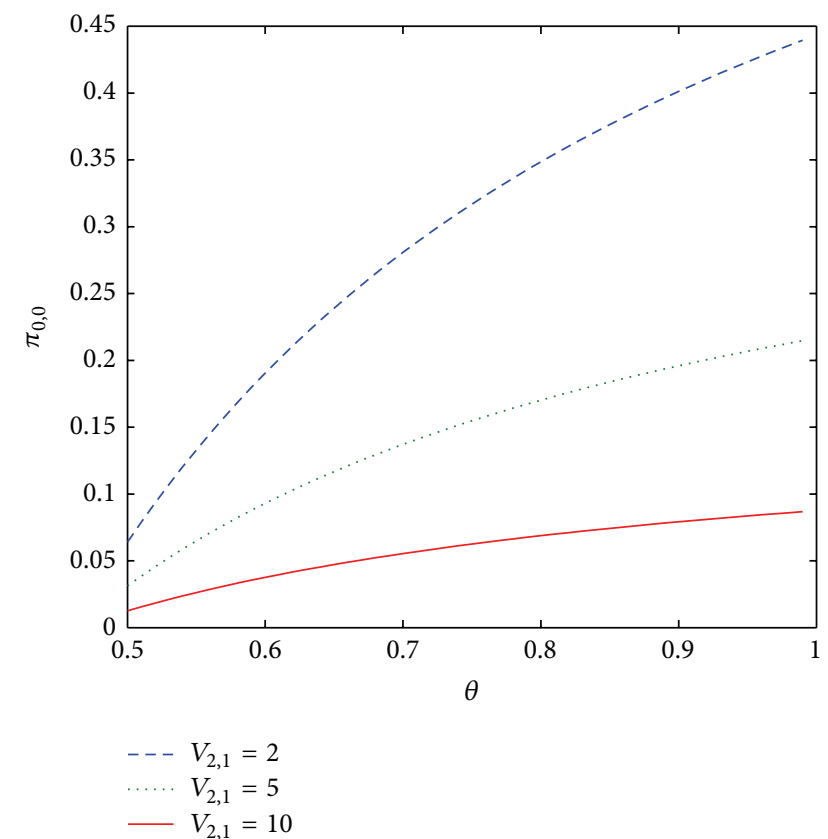

Figure $2: \pi_{0,0}$ versus $\theta$ for $p=0.2, r=0.1, \mu=0.3$, and $v_{1}=0.5$.

take exhaustive single vacation and nonexhaustive urgent vacation. We firstly analyze the Markov chain underlying the considered queueing system and present some performance measures of the system such as the generating functions of system state distribution, the mean orbit size, and system size. Secondly, a stochastic decomposition result and the relationship between our model and the corresponding continuous-time model are given. Finally, we show the effects of different parameters on some of the main performance

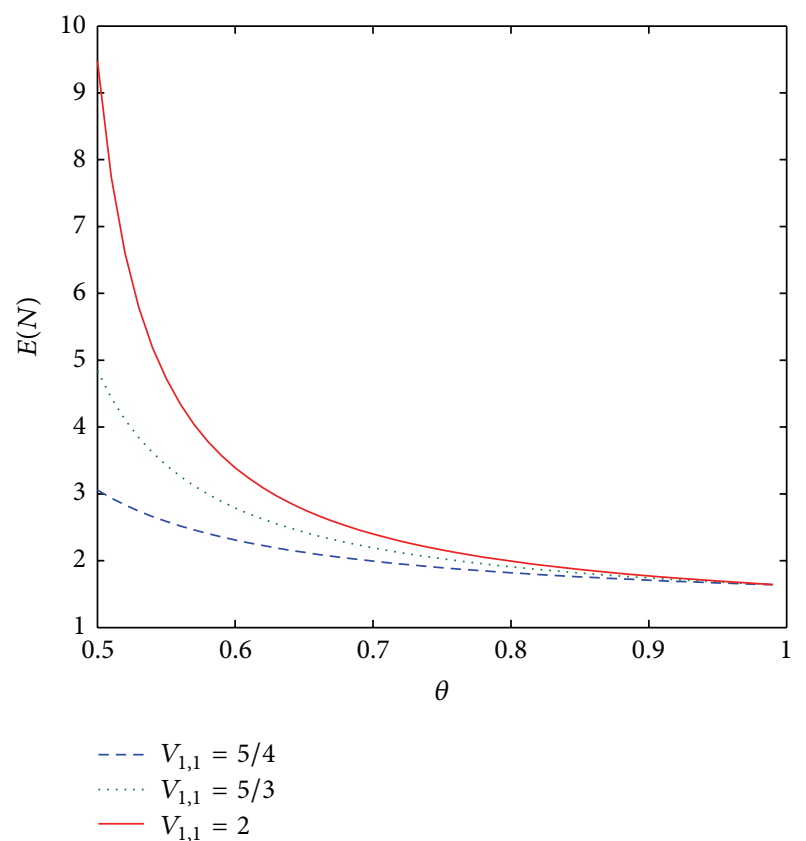

FIGURE 3: $E(N)$ versus $\theta$ for $p=0.2, r=0.1, \mu=0.3$, and $v_{1}=0.5$.

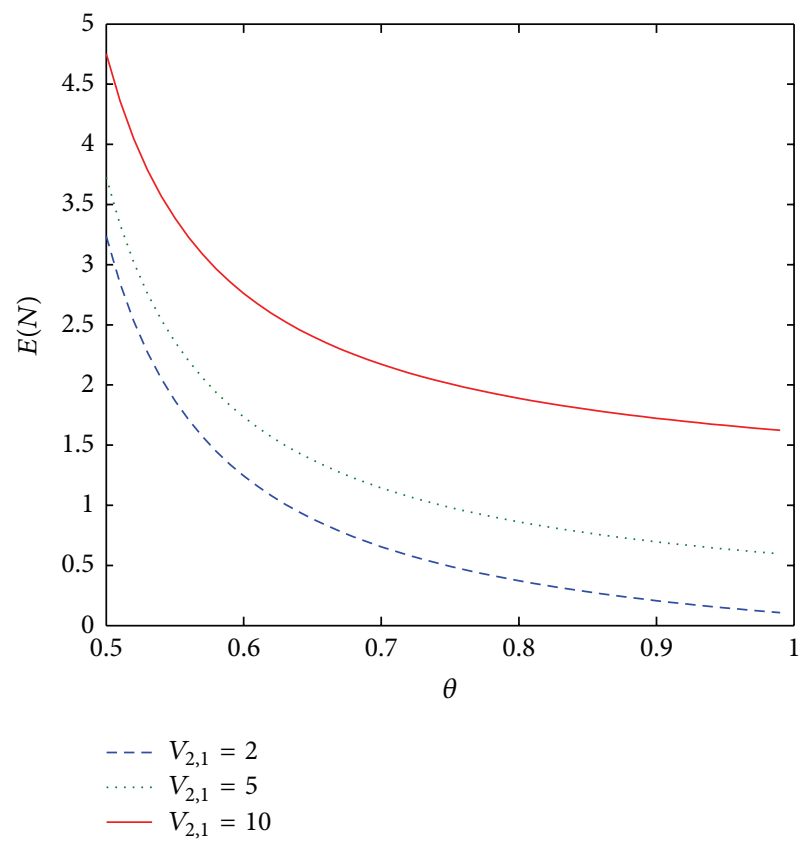

Figure 4: $E(N)$ versus $\theta$ for $r=0.1, \mu=0.3, p=0.2$, and $v_{2}=0.9$.

measures through some numerical examples. The waiting time and busy period in our model are quite difficult to obtain due to the possible nonexhaustive vacations of the server. In case of server's nonexhaustive vacation, the service process of a customer may be interrupted and the customer enters the orbit. This complicates the analysis of waiting time distribution of a tagged customer in the orbit. Thus, the waiting time distribution and the busy period deserve further investigation in the future. 


\section{Appendix}

\section{Proof of Lemma 2}

Proof. Define the functions $f(z)=\bar{p} A(\bar{p})(1-z) \Omega(z)+$ $z \theta \gamma^{2}(z)$ and $g(z)=z \gamma(z)$ for $0 \leq z<1$. It is easy to verify that $f^{\prime}(1)=(1-S(\theta))\left(p \theta+\bar{\theta}+p V_{1,1} \bar{\theta}\right)-1-p \theta-\bar{\theta} \bar{p} A(\bar{p})$ and $g^{\prime}(1)=1+p$. Thus $f^{\prime}(1)<g^{\prime}(1)$ is equivalent to $p \theta+\bar{\theta}+\bar{\theta} p V_{1,1}<\rho_{1}$. Since $f^{\prime \prime}(z)=[1-\bar{p} A(\bar{p})] \Omega(z)+$ $[z+\bar{p} A(\bar{p})(1-z)] \Omega^{\prime}(z)+\theta \gamma^{2}(z)+2 p \theta z \gamma(z)>0$ and $g^{\prime \prime}(z)=2 p>0$, we have that $f(z)$ and $g(z)$ are convex. So, we have $f(z)>g(z)$ for $0 \leq z \leq 1$.

Remark A.1. Let $\pi_{0,0}>0$; we obtain that $p \theta+\bar{\theta}+\bar{\theta} p V_{1,1}<\rho_{1}$ is a necessary condition for the stability of our system. When we consider the special case that "the server can not take urgent vacation" the above condition can be rewritten as $p S_{1}-p<$ $\bar{p} A(\bar{p})$, where the left-hand side is the expected number of external customers who arrive per service interval and the right-hand side represents the expected number of repeated customers who enter service at the epoch at which a service starts on average. This condition agrees with the result in the discrete-time general retrial queue with exhaustive vacation.

\section{Conflict of Interests}

The authors declare that there is no conflict of interests regarding the publication of this paper.

\section{References}

[1] T. Yang and J. G. C. Templeton, "A survey of retrial queues," Queueing Systems, vol. 2, no. 3, pp. 201-233, 1987.

[2] A. Gomez-Corral, "A bibliographical guide to the analysis of retrial queues through matrix analytic techniques," Annals of Operations Research, vol. 141, pp. 163-191, 2006.

[3] G. I. Falin, "A survey of retrial queues," Queueing Systems. Theory and Applications, vol. 7, no. 2, pp. 127-167, 1990.

[4] J. R. Artalejo and A. Gomez-Corral, Retrial Queueing Systems: A Computational Approach, Springer, Berlin, Germany, 2008.

[5] J. R. Artalejo, "Accessible bibliography on retrial queues: progress in 2000-2009," Mathematical and Computer Modelling, vol. 51, no. 9-10, pp. 1071-1081, 2010.

[6] H. Li and T. Yang, "A single-server retrial queue with server vacations and a finite number of input sources," European Journal of Operational Research, vol. 85, no. 1, pp. 149-160, 1995.

[7] B. K. Kumar and D. Arivudainambi, "The $M / G / 1$ retrial queue with Bernoulli schedules and general retrial times," Computers \& Mathematics with Applications, vol. 43, no. 1-2, pp. 15-30, 2002.

[8] B. K. Kumar, R. Rukmani, and V. Thangaraj, "An M/M/C retrial queueing system with Bernoulli vacations," Journal of Systems Science and Systems Engineering, vol. 18, no. 2, pp. 222-242, 2009.

[9] J. R. Artalejo, "Analysis of an $M / G / 1$ queue with constant repeated attempts and server vacations," Computers \& Operations Research, vol. 24, no. 6, pp. 493-504, 1997.
[10] A. Aissani, "An $M^{X} / G / 1$ energetic retrial queue with vacations and it's control," Electronic Notes in Theoretical Computer Science, vol. 253, no. 3, pp. 33-44, 2009.

[11] F.-M. Chang and J.-C. Ke, "On a batch retrial model with $J$ vacations," Journal of Computational and Applied Mathematics, vol. 232, no. 2, pp. 402-414, 2009.

[12] J.-C. Ke and F.-M. Chang, "Modified vacation policy for $M / G / 1$ retrial queue with balking and feedback," Computers and Industrial Engineering, vol. 57, no. 1, pp. 433-443, 2009.

[13] T. Yang and H. Li, "On the steady-state queue size distribution of the discrete time $G e o / G / 1$ queue with repeated customers," Queueing Systems, vol. 21, no. 1-2, pp. 199-215, 1995.

[14] I. Atencia and P. Moreno, "A discrete-time Geo/G/1 retrial queue with general retrial times," Queueing Systems, vol. 48, no. 1-2, pp. $5-21,2004$.

[15] J. Wang and Q. Zhao, "Discrete-time Geo/G/1 retrial queue with general retrial times and starting failures," Mathematical and Computer Modelling, vol. 45, no. 7-8, pp. 853-863, 2007.

[16] I. Atencia, I. Fortes, and S. Sánchez, "A discrete-time retrial queueing system with starting failures, Bernoulli feedback and general retrial times," Computers and Industrial Engineering, vol. 57, no. 4, pp. 1291-1299, 2009.

[17] A. K. Aboul-Hassan, S. I. Rabia, and F. A. Taboly, "A discrete time $G e o / G / 1$ retrial queue with general retrial times and balking customers," Journal of the Korean Statistical Society, vol. 37, no. 4, pp. 335-348, 2008.

[18] A. K. Aboul-Hassan, S. I. Rabia, and F. A. Taboly, "Performance evaluation of a discretetime $G e o^{X} / G / 1$ retrial queue with general retrial times," Computers and Mathematics with Applications, vol. 58, pp. 548-557, 2009.

[19] J. B. Wu, Z. M. Liu, and Y. Peng, "A discrete-time Geo/G/1 retrial queue with preemptive resume and collisions," Applied Mathematical Modelling, vol. 35, no. 2, pp. 837-847, 2011.

[20] J. Wang, "Discrete-time Geo/G/1 retrial queues with general retrial time and Bernoulli vacation," Journal of Systems Science \& Complexity, vol. 25, no. 3, pp. 504-513, 2012.

[21] F. Zhang, D. Yue, and Z. Zhu, "A discrete time Geo/G/1 retrial queue with single vacation and starting failure," Journal of Information and Computational Science, vol. 8, no. 13, pp. 27512758, 2011.

[22] D. Yue and F. Zhang, "A discrete-time Geo/G/1 retrial queue with J-vacation policy and general retrial times," Journal of Systems Science \& Complexity, vol. 26, no. 4, pp. 556-571, 2013.

[23] F. Zhang and Z. Zhu, "A discrete-time Geo/G/1 retrial queue with $J$ vacations and two types of breakdowns," Journal of Applied Mathematics, vol. 2013, Article ID 834731, 11 pages, 2013.

[24] J. B. Wu and X. L. Yin, "An $M / G / 1$ retrial G-Queue with non-exhaustive random vacations and an unreliable server," Computers \& Mathematics with Applications, vol. 62, no. 5, pp. 2314-2329, 2011.

[25] Z. Zhou and Y. Zhu, "Optimization of the $\left(M A P_{1}, M A P_{2}\right) /\left(P H_{1}\right.$, $\left.\mathrm{PH}_{2}\right) / \mathrm{N}$ retrial queue model of wireless cellular networks with channel allocation," Computers and Electrical Engineering, vol. 39, no. 6, pp. 1637-1649, 2013.

[26] J. R. Artalejo and M. J. Lopez-Herrero, "Cellular mobile networks with repeated calls operating in random environment," Computers \& Operations Research, vol. 37, no. 7, pp. 1158-1166, 2010.

[27] T. V. Do, "Solution for a retrial queueing problem in cellular networks with the Fractional Guard Channel policy," Mathematical and Computer Modelling, vol. 53, no. 11-12, pp. 2059-2066, 2011. 
[28] B. D. Choi, K. K. Park, and C. E. M. Pearce, "An $M / M / 1$ retrial queue with control policy and general retrial times," Queueing Systems, vol. 14, no. 3-4, pp. 275-292, 1993.

[29] K. Avrachenkov and U. Yechiali, "On tandem blocking queues with a common retrial queue," Computers \& Operations Research, vol. 37, no. 7, pp. 1174-1180, 2010.

[30] K. Avrachenkov and U. Yechiali, "Retrial networks with finite buffers and their application to internet data traffic," Probability in the Engineering and Informational Sciences, vol. 22, no. 4, pp. 519-536, 2008.

[31] S. W. Fuhrmann and R. B. Cooper, "Stochastic decompositions in the $M / G / 1$ queue with generalized vacations," Operations Research, vol. 33, no. 5, pp. 1117-1129, 1985.

[32] J. R. Artalejo and J. I. Falin, "Stochastic decomposition for retrial queues," Top, vol. 2, no. 2, pp. 329-342, 1994. 


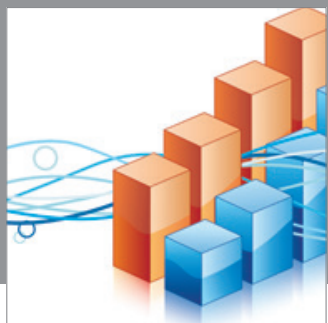

Advances in

Operations Research

mansans

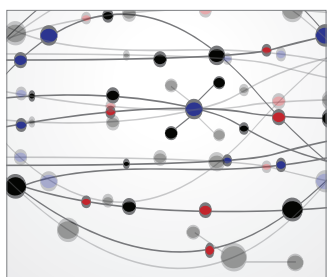

The Scientific World Journal
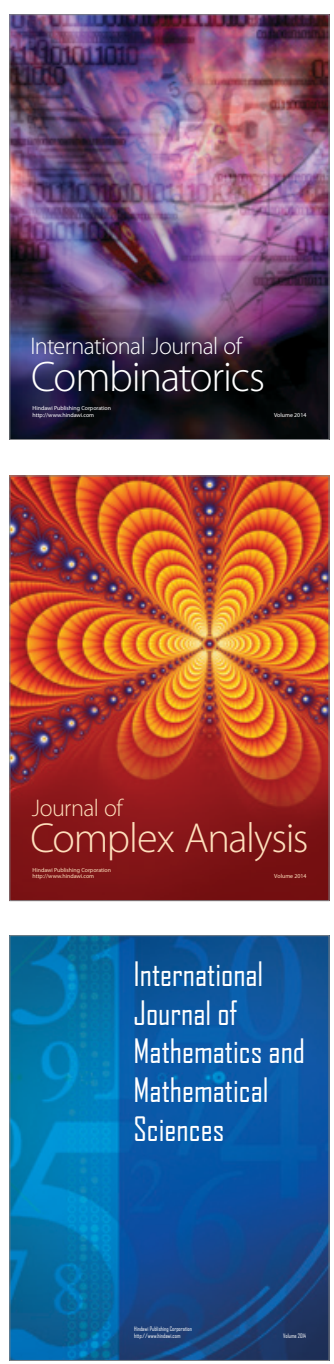
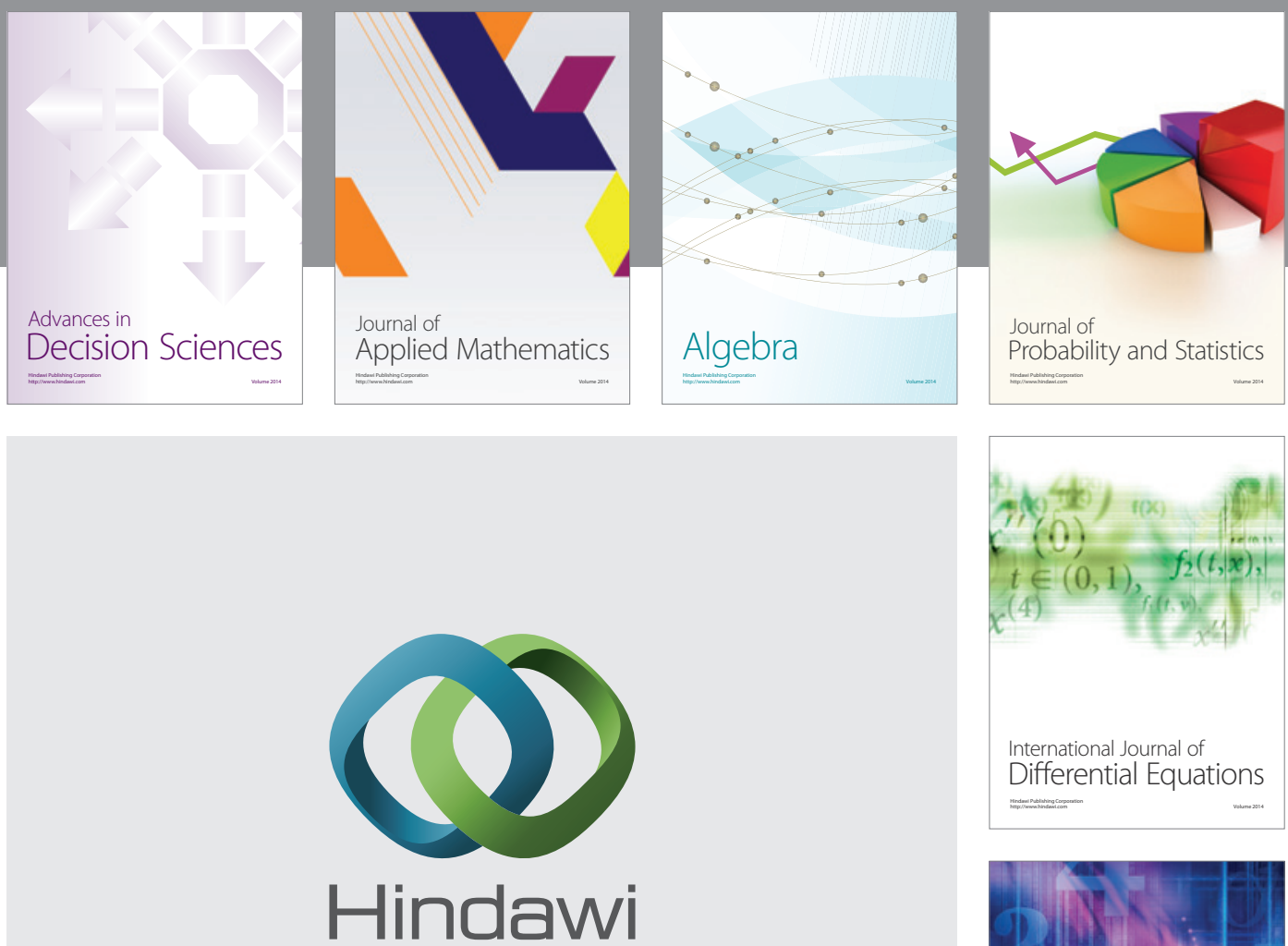

Submit your manuscripts at http://www.hindawi.com
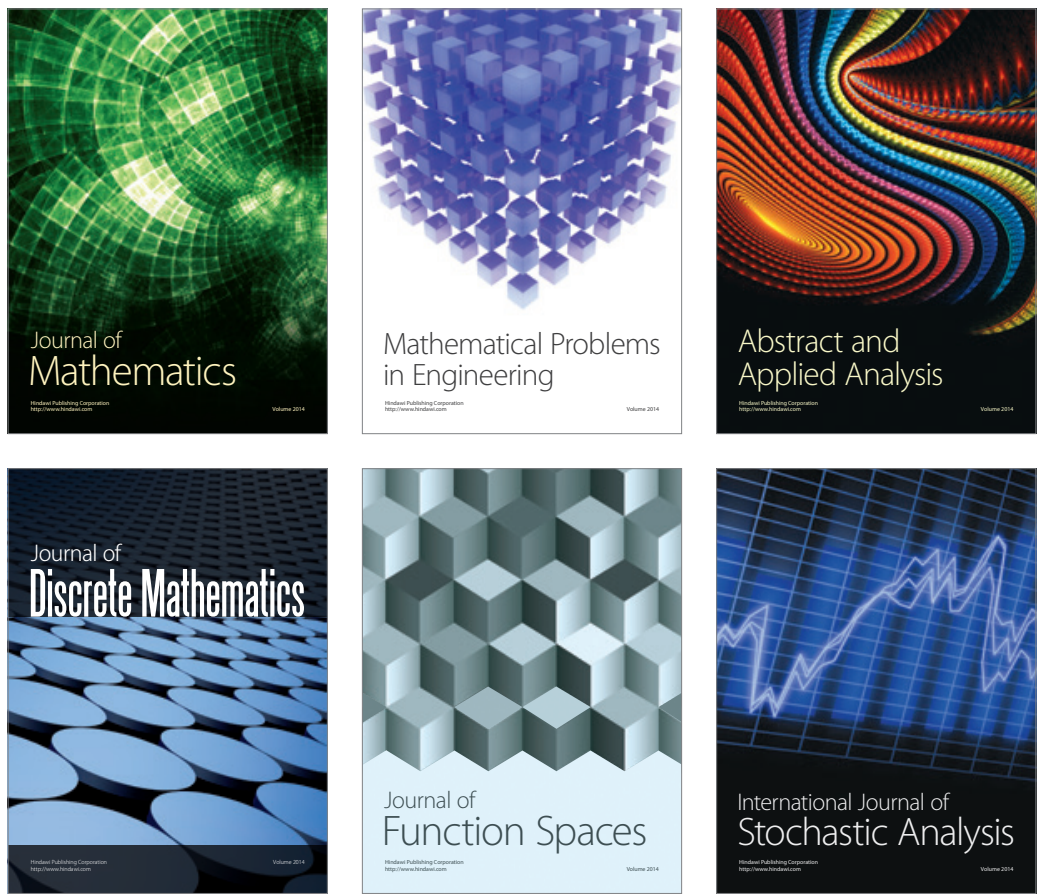

Journal of

Function Spaces

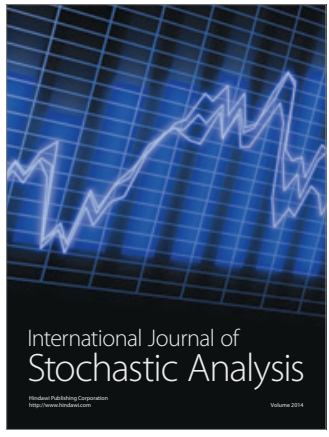

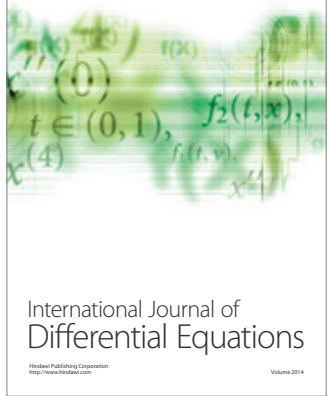
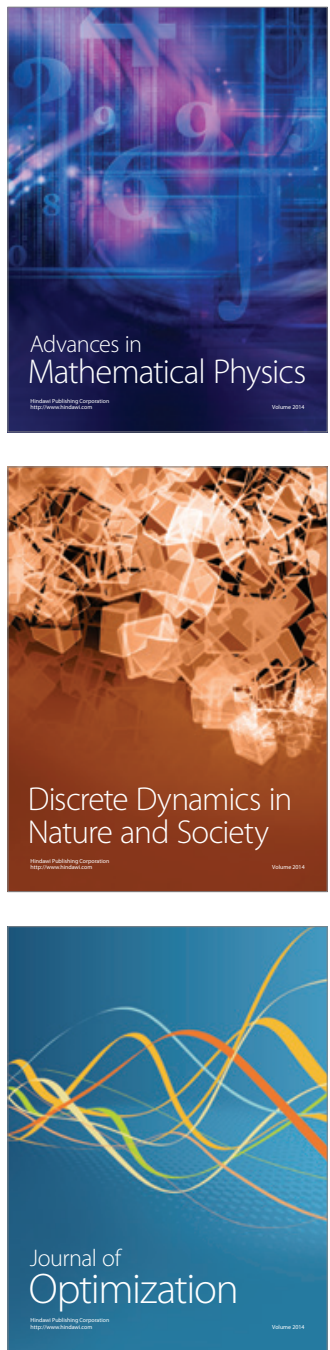